\title{
miR-502-5p inhibits the proliferation, migration and invasion of gastric cancer cells by targeting SP1
}

\author{
XIAOBO PENG $^{1 *}$, MEIHONG WU $^{1 *}$, WUXIA LIU ${ }^{2}$, CHENGTAO GUO $^{1}$, LIXING ZHAN $^{3}$ and XIANBAO ZHAN ${ }^{1}$ \\ ${ }^{1}$ Department of Oncology; ${ }^{2}$ VIP Clinic, Changhai Hospital, Naval Military Medical University, \\ Shanghai 200433; ${ }^{3}$ Key Laboratory of Food Safety Research, Institute for Nutritional Sciences, \\ Shanghai Institute of Biological Sciences, Chinese Academy of Sciences, Shanghai 200031, P.R. China
}

Received August 26, 2018; Accepted June 8, 2020

DOI: $10.3892 / \mathrm{ol} .2020 .11808$

\begin{abstract}
Gastric cancer (GC) is the third most common cause of cancer-associated mortality in China. Aberrant microRNA (miR) expression can occur through multiple biological processes and has been implicated in cancer development. However, to the best of our knowledge, the function of miR-502-5p in GC is currently unclear. In the present study, the expression and function of miR-502-5p in GC was evaluated. Reverse transcription-quantitative (RT-q) PCR was used to measure the expression levels of miR-502-5p in GC tissues, normal adjacent tissues, a normal human gastric epithelial cell line (GES-1) and two GC cell lines. miR-502-5p expression levels were significantly lower in GC tissues and GC cell lines compared with those in adjacent normal tissues and GES-1 cells, respectively. Subsequently, the target genes of miR-502-5p were predicted, and it was demonstrated that the transcription factor SP1 was a direct target. SP1 expression, cell viability, migration and invasion, and SP1 protein levels were examined using RT-qPCR, an MTT assay, Transwell assay and western blotting, respectively. Human GC cells were then transfected with an miR-502-5p mimic to emulate miR-502-5p overexpression, resulting in inhibition of the proliferation, migration and invasion capacities of human GC cells. Compared with the negative control, cells overexpressing miR-502-5p had decreased levels of SP1 mRNA and protein. These data suggest that miR-502-5p
\end{abstract}

Correspondence to: Professor Xianbao Zhan, Department of Oncology, Changhai Hospital, Naval Military Medical University, 168 Chang Hai Road, Shanghai 200433, P.R. China

E-mail: zhanxianbaoch@163.com

Professor Lixing Zhan, Key Laboratory of Food Safety Research, Institute for Nutritional Sciences, Shanghai Institute of Biological Sciences, Chinese Academy of Sciences, 320 Yue Yang Road, Shanghai 200031, P.R. China

E-mail:361579491@qq.com

${ }^{*}$ Contributed equally

Key words: microRNA-502-5p, proliferation, migration, invasion, gastric cancer, SP1 serves as a tumor suppressor gene by targeting SP1 to regulate the proliferation, migration and invasion of GC cells.

\section{Introduction}

Gastric cancer (GC) is the second most commonly diagnosed cancer among men and the third among women, and it was the second leading cause of cancer-associated death worldwide in 2015 (1). Due to the lack of specific early symptoms, the majority of patients are not diagnosed until they have advanced stage GC. The overall prognosis of patients with GC is poor and the 5-year survival rate is $<30 \%(2-4)$. GC is a heterogeneous disease characterized by different molecular and histological profiles (5), therefore it is important to identify novel sensitive and specific biomarkers for early diagnosis. In addition, a more comprehensive understanding of tumor suppressor genes may provide novel insight into GC therapeutics.

MicroRNAs (miRNAs/miRs) are a conserved group of single-stranded non-coding RNAs that are 17-25 nucleotides long (6). miRNAs directly bind to the 3'-untranslated region of their target mRNA to regulate gene expression after transcription, thereby inhibiting translation or inducing mRNA degradation (7). miRNAs are involved in carcinogenesis, including tumor initiation and disease progression $(8,9)$. In cancer, miRNAs can function as oncogenes or tumor suppressors depending on the function of its target gene $(10,11)$. Recent studies have demonstrated that several miRNAs are involved in tumor occurrence and can function as oncogenes or tumor suppressor genes in GC (12-14). For example, miR-6852 functions as a tumor suppressor by directly targeting forkhead box $\mathrm{J} 1$ in gastric cancer (13). In addition, by targeting the B cell lymphoma-2 gene, miR-744 can promote apoptosis in the GC cell line SGC-7901 (14). Therefore, it is important to explore the molecular mechanisms underlying miRNA function in GC to promote the development of targeted therapies.

The purpose of the present study was to investigate the expression levels and function of miR-502-5p and its molecular mechanisms in GC.

\section{Materials and methods}

Tissue samples, cells and reagents. Between July 2017 and February 2018, 32 samples of GC and adjacent tissues (5 $\mathrm{cm}$ away from tumor tissue) were obtained from patients, 
including 19 male and 13 female subjects, and the median age was 57 years (age range, 35-84 years). All patients underwent gastrectomy at the Changhai Hospital of Naval Medical University (Shanghai, China). Tissue samples from the patients with GC were immediately flash frozen in liquid nitrogen following resection at $-196^{\circ} \mathrm{C}$. The Changhai Hospital Ethics Committee (Shanghai, China) approved the present study and all patients provided written informed consent.

AGS and MKN45 human GC cells and GES human normal gastric cells were purchased from the American Type Culture Collection. AGS, MKN45 and GES-1 cells were cultured in RPMI-1640 medium (Hyclone; GE Healthcare Life Sciences) containing $10 \%$ fetal bovine serum (Hyclone; GE Healthcare Life Sciences), $100 \mathrm{IU} / \mathrm{ml}$ penicillin and $100 \mu \mathrm{g} / \mathrm{ml}$ streptomycin.

microRNA and cell transfection. miR-502-5p mimic and miR-502-5p-mimic negative control (NC) was purchased from Shanghai GenePharma Co., Ltd. The microRNA was transfected into GC cells using Lipofectamine 2000 reagent (Thermo Fisher Scientific Inc.) according to the manufacturer's protocol. The time interval between transfection and subsequent experimentation was $48 \mathrm{~h}$.

$R N A$ and reverse transcription-quantitative $(R T-q) P C R$. Total RNA was extracted from the patient tissue samples, and AGS, MKN45 and GES cells using TRIzol ${ }^{\circledR}$ reagent (Invitrogen; Thermo Fisher Scientific Inc.). miR-502-5p expression levels were measured using a TaqMan microRNA assay kit (Takara Bio. Inc.) according to the manufacturer's instructions, using U6 as an internal control. Total RNA was then reverse transcribed into cDNA using a Prime Script RT reagent kit (Takara Bio. Inc.) according to the manufacturer's instructions. SYBR-Green (Takara Bio. Inc.) was used to determine SP1 mRNA expression relative to $\beta$-actin. The thermocycling conditions for qPCR were as follows: $95^{\circ} \mathrm{C}$ for $5 \mathrm{~min}$ followed by 40 cycles of $95^{\circ} \mathrm{C}$ for $10 \mathrm{sec}, 60^{\circ} \mathrm{C}$ for $30 \mathrm{sec}$. The primers were designed as follows: miR-502-5p forward, 5'-CGGGCATCCTTGCTATCTG-3' and reverse, 5'-CAGCCACAAAAGAGCACAAT-3'; U6 forward, 5'-CTC GCTTCGGCAGCACA-3' and reverse, 5'-AACGCTTCACGA ATTTGCGT-3'; SP1 forward, 5'-TGGCAGCAGTACCAA TGGC-3' and reverse, 5'-CCAGGTAGTCCTGTCAGAACT T-3'; and $\beta$-actin forward, 5'-CCTGGCACCCAGCACAAT-3' and reverse: 5'-GGGCGGGACTCGTCATAC-3'. Each sample was analyzed in triplicate and levels were quantified using the $2^{-\Delta \Delta \mathrm{Cq}}$ method (15).

MTT cell proliferation assay. AGS and MKN45 cells were transfected with miR-502-5p mimics or NC for $24 \mathrm{~h}$ as aforementioned. AGS and MKN45 cells were collected and $5 \times 10^{3}$ cells per well were seeded into 96 -well plates in triplicate. Following 1-4 days in the incubator at $37^{\circ} \mathrm{C}$ with an atmosphere of $5 \% \mathrm{CO}_{2}, 10 \mu \mathrm{l}$ MTT assay solution was added to each well for $4 \mathrm{~h}$ at $37^{\circ} \mathrm{C}$. Next, $100 \mu \mathrm{l}$ DMSO was added to each well for $30 \mathrm{~min}$ to dissolve the purple formazan, and optical density was measured at $490 \mathrm{~nm}$ with a microplate reader (Bio-Rad Laboratories, Inc.).

Migration and invasion Transwell assays. In the migration assay, $1 \times 10^{5}$ cells were plated in $200 \mu 1$ serum-free medium in the top Transwell chamber. In the invasive assay, $1 \times 10^{5}$ cells were plated in $200 \mu \mathrm{l}$ serum-free medium in the top Transwell chamber with a Matrigel-coated membrane. The matrigel was pre-coated at $37^{\circ} \mathrm{C}$ for $30 \mathrm{~min}$. In both the migration and invasion experiments, $500 \mu \mathrm{l}$ medium containing $10 \%$ FBS was added into the lower chamber as a chemoattractant. After $24 \mathrm{~h}$, the cells on the top surface of the Transwell chamber were removed using cotton swabs. The cells on the bottom surface were fixed at room temperature with $100 \%$ methanol for $30 \mathrm{~min}$, and then stained with $0.05 \%$ crystal violet for $30 \mathrm{~min}$ at room temperature. Five visual fields were randomly selected to photograph with an Olympus IX51 light microscope (Olympus Corporation; magnification, x20).

Western blot analysis. Transfected AGS and MKN45 cells were lysed with RIPA buffer (Cell Signaling Technology, Inc.) containing complete protease inhibitor cocktail (Roche Diagnostics), phosphatase inhibitors (Roche Diagnostics), $5 \mathrm{mM}$ dithiothreitol (DTT, Sigma-Aldrich; Merck KGaA) and $1 \mathrm{mM}$ phenyl methyl sulfonyl fluoride (Sigma-Aldrich; Merck KGaA). The supernatant of the cell lysate was collected and protein concentrations were determined using the bicinchoninic protein assay kit (Thermo Fisher Scientific Inc.) according to the manufacturer's instructions. Then $20 \mu \mathrm{g}$ protein was loaded onto a $10 \%$ gel, resolved using SDS-PAGE and transferred onto PVDF membranes. Membranes were then blocked with 5\% fat-free milk for $2 \mathrm{~h}$ at room temperature. Subsequently, membranes were incubated with primary antibodies against SP1 (1:1,000; cat. no. WL02251; Wanleibio Co., Ltd.) and $\beta$-actin (1:1,000; cat. no. P30002M; Abmart Pharmaceutical Technology Co., Ltd.) at $4^{\circ} \mathrm{C}$ overnight, washed three times with TBST (0.05\% Tween-20) and incubated with anti-mouse horseradish peroxidase-conjugated secondary antibody (1:2,000; cat. no. 7054S; Cell Signaling Technology, Inc.) at room temperature for $2 \mathrm{~h}$. Following three washes with TBST, immunoreactive bands were visualized using ECL working fluid (Biochannel, Nanjing, China; http: //www. biochannel.cn/page19.html?product_id=299). This experiment was repeated three times.

Identification of miR-502-5p target genes. Target Scan version.7.2(http://www.targetscan.org/), miRandaversion.2010 (http: //www.microrna.org/microrna/getGeneForm.do) and miRBase version.22.1 (http: //www.mirbase.org/) were used to predict the candidate target genes of miR-502-5p.

Statistical analysis. All data are expressed as the mean \pm standard deviation, and statistical analyses were performed using SPSS version 17.0 (SPSS, Inc.). Student's paired t-tests were used for comparisons between two groups and one-way ANOVA followed by Tukey's post hoc test was used for multiple comparisons. $\mathrm{P}<0.05$ was considered to indicate a statistically significant difference.

\section{Results}

Expression of miR-502-5p in GC tissues and cells. Expression levels of miR-502-5p in $32 \mathrm{GC}$ tissues and two GC cell lines were analyzed using RT-qPCR. The expression level of miR-502-5p in GC tissues was significantly lower compared with those in matched normal adjacent tissues $(\mathrm{P}<0.05$; 
A

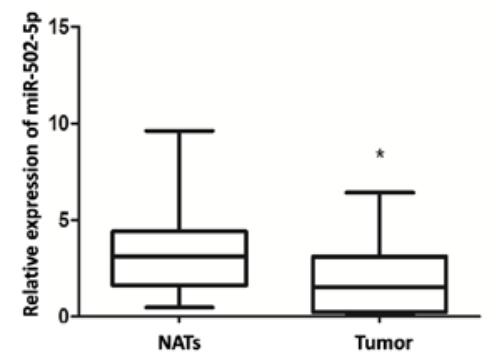

B

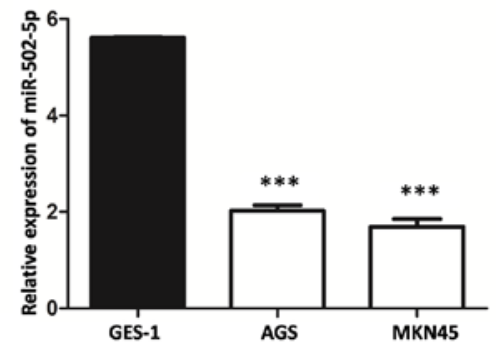

Figure 1. Expression of in miR-502-5p gastric cancer tissues and cells. (A) Expression of miR-502-5p in gastric cancer tissues compared with NATs "P<0.05 vs. NATs. (B) Expression of miR-502-5p in GC cells compared with GES-1 cells. ${ }^{* * *} \mathrm{P}<0.001$ vs. GES-1. NATs, normal adjacent tissues; miR-502-5p, microRNA-502-5p.

A

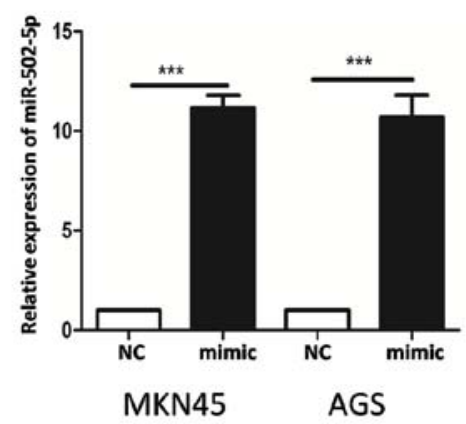

B

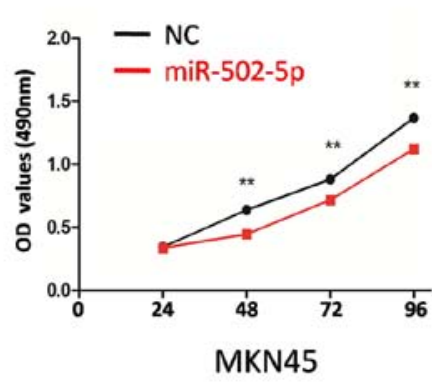

D
C

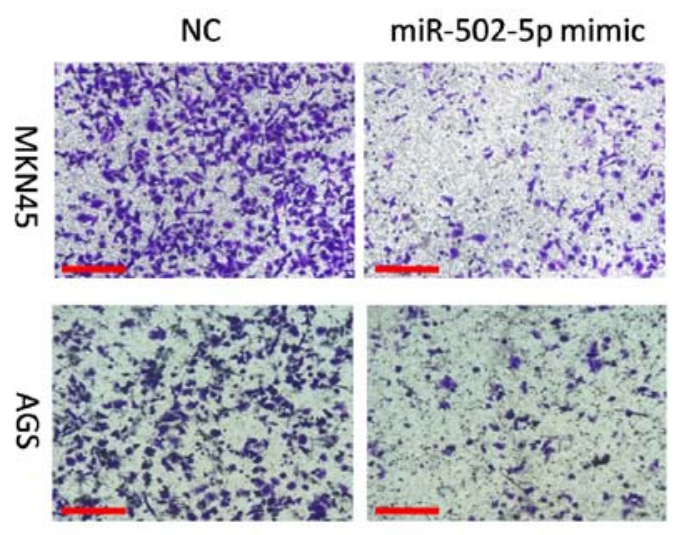

NC

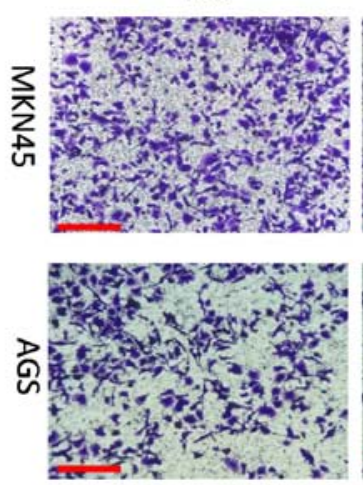

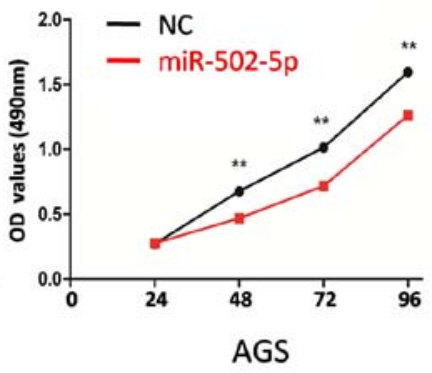

AGS
miR-502-5p mimic
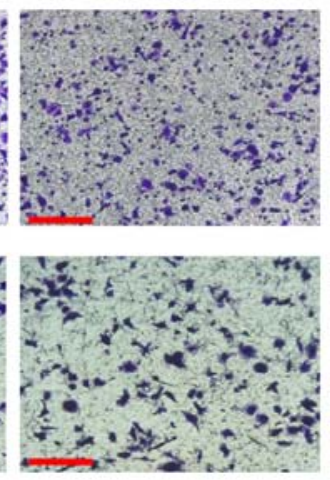

Figure 2. miR-502-5p inhibits the proliferation, migration and invasion of MKN45 and AGS cells. (A) miR-502-5p expression was evaluated in MKN45 and AGS cells transfected with miR-502-5p mimic or NC. ${ }^{* * *} \mathrm{P}<0.001$. (B) Effect of miR-502-5p on the proliferation of MKN45 and AGS cells was determined using an MTT assay. ${ }^{* *} \mathrm{P}<0.01$ vs. miR-502-5p mimic. Effects of miR-502-5p on the (C) migration and (D) invasion of MKN45 and AGS cells were determined using a Transwell assay. Scale bar=200 $\mu \mathrm{m}$. miR, microRNA; NC, negative control; OD, optical density.

Fig. 1A). Compared with normal GES-1 gastric epithelial cells, the two GC cell lines exhibited significantly lower miR-502-5p expression ( $\mathrm{P}<0.001$; Fig. 1B).

miR-502-5p inhibits the proliferation, migration and invasion of GC cells. To study the effect of miR-502-5p on GC cells, $\mathrm{NC}$ or mimics of miR-502-5p were transfected into MKN45 and AGSGC cells. The transfection efficiency of miR-502-5p was evaluated using RT-qPCR. The level of miR-502-5p in MKN45 and AGS cells transfected with miR-502-5p mimics was significantly higher compared with those transfected with NC (both P<0.001; Fig. 2A). Overexpression of miR-502-5p significantly decreased proliferation at all time points compared with the $\mathrm{NC}$ group (all $\mathrm{P}<0.01$; Fig. 2B) and reduced the cellular migration and invasion capacities of MKN45 and AGSGC cells (Fig. 2C and D).

miR-502-5p targets $S P 1$. To investigate the molecular mechanism underlying miR-502-5p-mediated inhibition of GC progression, target genes of miR-502-5p were predicted using miRNA prediction software and databases. Target Scan 7.2, miR and a 2010 and miR Base 22.1 predicted that SP1 is a target gene of miR-502-5p. The mRNA level of SP1 decreased significantly in AGS and MKN45 cells transfected with miR-502-5p mimic $(\mathrm{P}<0.05$ 
A

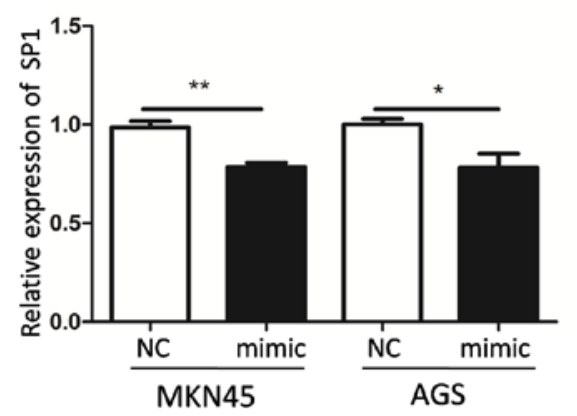

C

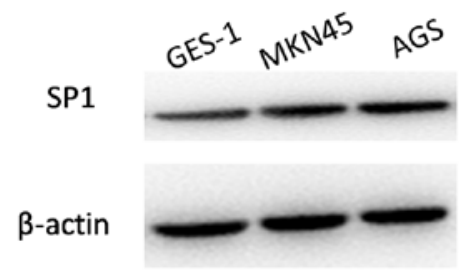

B

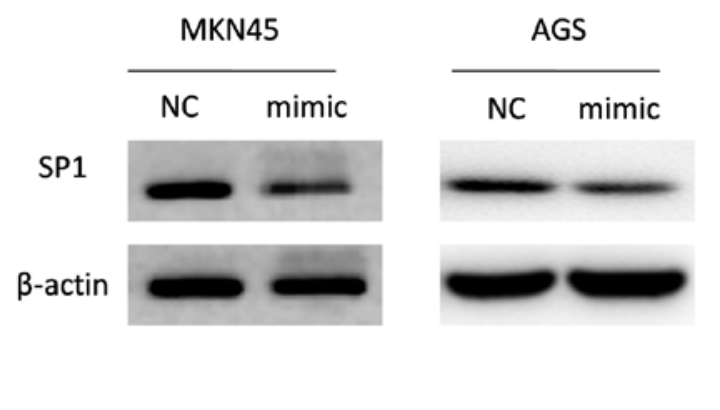

D

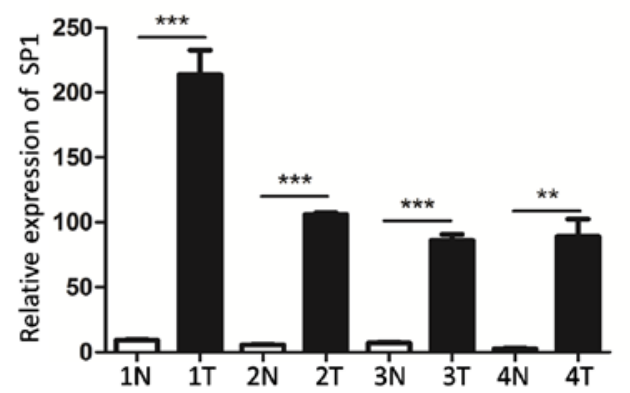

Figure 3. miR-502-5p targets SP1. (A) Expression of SP1 mRNA in AGS and MKN45 cells transfected with miR-502-5p mimic or NC was detected using reverse transcription quantitative-PCR. (B) Expression of SP1 protein in AGS and MKN45 cells transfected with miR-502-5p mimic or NC was analyzed using western blotting. (C) Expression of SP1 protein was evaluated in AGS and MKN45 gastric cancer cell lines and the GES-1 cell line. (D) Expression of SP1 mRNA was evaluated in four gastric cancer tissue samples. ${ }^{*} \mathrm{P}<0.05,{ }^{* *} \mathrm{P}<0.01,{ }^{* * *} \mathrm{P}<0.001$. miR, microRNA; NC, negative control; $\mathrm{T}$, tumor; $\mathrm{N}$, normal.

and $\mathrm{P}<0.01$, respectively, Fig. 3A). In addition, western blotting demonstrated that the protein level of SP1 was lower in AGS and MKN45 cells transfected with miR-502-5p mimics compared with the controls. SP1 levels were also evaluated in GC cells. Compared with GES cells, the expression of miR-502-5p in AGS and MKN45 cells was lower (Fig. 1B), but the expression of SP1 was higher (Fig. 3C). The mRNA expression level of SP1 in four sets of tumor tissues was significantly higher compared with that in normal paracancerous tissues (Fig. 3D). This suggests that SP1 is targeted by miR-502-5p in GC.

\section{Discussion}

In GC, miRNAs are considered as novel potential diagnostic biomarkers, prognostic factors and therapeutic targets (16). Abnormal expression of miRNAs in GC has previously been reported $(13,14)$. However, the specific role and subsequent underlying molecular mechanisms of miR-502-5p in GC remain unclear. To the best of our knowledge, the present study is the first to report the expression, biological function and molecular mechanism of miR-502-5p in GC. The level of miR-502-5p was downregulated in GC tissues and cell lines, and, in functional analyses, miR-502-5p significantly decreased the proliferative, migratory and invasive properties of GC cells.

Aberrant expression of miR-502-5p is associated with tumorigenesis and is a tumor suppressor in hepatocellular carcinoma, breast and colon cancer (17-19). In hepatocellular carcinoma cells, miR-502-5p significantly inhibits proliferation invitro and tumor growth invivo by targetingphophatidylinositor-4, 5-bisphosphate 3-kinase catalytic subunit $\mathrm{y}$ (17). It has been reported that miR-502-5p expression in MCF-7 and MDA-MB-231 cells is low, and miR-502-5p can promote apoptosis and inhibit the proliferation of breast cancer cells in vitro by binding to the tumor necrosis factor receptor-associated factor 2 (TRAF2) gene in breast cancer (18). Another study indicated that miR-502 can inhibit autophagy, proliferation and cell cycle progression in colon cancer cells in vitro. Furthermore, miR-502 can inhibit colon cancer progression in mouse tumor xenografts models in vivo (19). These previous studies suggest that miR-502 may be implicated in tumor progression.

SP1 is a ubiquitous transcription regulator in human cells that regulates proliferation, apoptosis and embryonic development (20). SP1 also promotes the invasion and metastasis of tumor cells by regulating cell adhesion protein matrix metalloproteinase, urokinase-type plasminogen activator and micro-vessel density in tumors (21). SP1 is abnormally expressed in gastric cancer cells and participates in the proliferation and apoptosis of these cells (22); however, the relationship between SP1 and tumor metastasis is complex. For example, in certain tumors, such as glioma (23) and colon cancer (24), the effect of SP1 on tumor metastasis can be reduced by inhibiting the expression of SP1 in tumor cells. However, in GC (25) and lung adenocarcinoma (26), inhibiting the expression of SP1 can promote the metastasis and invasion ability of tumor cells.

The present study investigated the molecular mechanisms underlying miR-502-5p function in GC. First, bioinformatics was used to predict the potential target genes of miR-502-5p, which identified SP1 as a candidate. Then, mRNA expression levels of SP1 were measured in four GC tissue sets. It was determined that SP1 mRNA levels were significantly higher 
compared with those in normal adjacent tissues. In addition, RT-qPCR and western blotting demonstrated that overexpression of miR-502-5p decreased the expression levels of SP1 mRNA and protein in GC cells, respectively. These results suggest that SP1 is a downstream target gene of miR-502-5p.

In conclusion, the present study demonstrated that miR-502-5p is a novel tumor suppressor, as overexpression of miR-502-5p inhibited the proliferation, migration and invasion of GC cells. Thus, downregulation of miR-502-5p may be necessary for GC carcinogenesis via SP1 regulation. The present findings may improve our understanding of the molecular pathogenesis of GC and highlight the potential of miR-502-5p as a target for antitumor therapy. However, the present study also has some limitations. For example, the effect of miR-502-5p on the biological behavior of GC cells at the cellular level was only investigated in vitro. In the future, further studies should be conducted to demonstrate the effect ofmiR-502-5p on the biological behavior of GC in vivo.

\section{Acknowledgements}

Not applicable.

\section{Funding}

The present study was funded by The National Natural Science Foundation of China (grant no. 81672892).

\section{Availability of data and materials}

The datasets used and/or analyzed during the current study are available from the corresponding author on reasonable request.

\section{Authors' contributions}

XP and MW designed the study. WL and CG performed the data analysis. CG sorted out the experimental data. XP, LZ and $\mathrm{XZ}$ performed the data analyses and wrote the manuscript. LZ taught the experimental protocols. All authors read and approved the final manuscript.

\section{Ethics approval and consent to participate}

The Changhai Hospital Ethics Committee (Shanghai, China) approved the present study (approval no. CHEC2016-157). All patients who agreed to participate in the study provided written informed consent.

\section{Patient consent for publication}

Not applicable.

\section{Competing interests}

The authors declare that they have no competing interests.

\section{References}

1. Chen W, Zheng R, Baade PD, Zhang S, Zeng H, Bray F, Jemal A, Yu XQ and He J: Cancer statistics in China, 2015. CA Cancer J Clin 66: 115-132, 2016.
2. Bria E, De Manzoni G, Beghelli S, Tomezzoli A, Barbi S, Di Gregorio C, Scardoni M, Amato E, Frizziero M, Sperduti I, et al: A clinical-biological risk stratification model for resected gastric cancer: Prognostic impact of Her2, Fhit, and APC expression status. Ann Oncol 24: 693-701, 2013.

3. Peng PL, Zhou XY, Yi GD, Chen PF, Wang F and Dong WG: Identification of a novel gene pairs signature in the prognosis of gastric cancer. Cancer Med 7: 344-350, 2018.

4. Akhondi-Meybodi M, Ghane M, Akhondi-Meybodi S and Dashti G: Five-year survival rate for gastric cancer in Yazd Province, Central Iran, from 2001 to 2008. Middle East J Dig Dis 9: 39-48, 2017.

5. Bass AJ, Thorsson V, Shmulevich I, Reynolds SM, Miller M, Bernard B, Hinoue T, Laird PW, Curtis C, Shen H, et al: Comprehensive molecular characterization of gastric adenocarcinoma. Nature 513: 202-209, 2014.

6. Bartel DP: MicroRNAs: Genomics, biogenesis, mechanism, and function. Cell 116: 281-297, 2004.

7. Ambros V: The functions of animal microRNAs. Nature 431: 350-355, 2004.

8. Lei T, Zhu Y, Jiang C, Wang Y, Fu J, Fan Z and Qin H: MicroRNA-320 was downregulated in non-small cell lung cancer and inhibited cell proliferation, migration and invasion by targeting fatty acid synthase. Mol Med Rep 14: 1255-1262, 2016.

9. Hagan JP and Croce CM: MicroRNAs in carcinogenesis. Cytogenet Genome Res 118: 252-259, 2007.

10. Xia E, Kanematsu S, Suenaga Y, Elzawahry A, Kondo H, Otsuka N, Moriya Y, Iizasa T, Kato M, Yoshino I and Yokoi S: MicroRNA induction by copy number gain is associated with poor outcome in squamous cell carcinoma of the lung. Sci Rep 8: 15363,2018

11. Kundu A, Quirit JG, Khouri MG and Firestone GL: Inhibition of oncogenic BRAF activity by indole-3-carbinol disrupts microphthalmia-associated transcription factor expression and arrests melanoma cell proliferation. Mol Carcinog 56: 49-61, 2017.

12. Ma X, Feng J, Lu M, Tang W, Han J, Luo X, Zhao Q and Yang L: microRNA-501-5p promotes cell proliferation and migration in gastric cancer by downregulating LPAR1. J Cell Biochem 121: 1911-1922, 2020

13. Yu H, Zhang J, Wen Q, Dai Y, Zhang W, Li F and Li J: MicroRNA-6852 suppresses cell proliferation and invasion via targeting forkhead box J1 in gastric cancer. Exp Ther Med 16: 3249-3255, 2018.

14. Liu J, Wei Y, Li S, Li Y, Liu H, Liu J and Zhu X: MicroRNA-744 promotes cell apoptosis via targeting $\mathrm{B}$ cell lymphoma-2 in gastric cancer cell line SGC-7901. Exp Ther Med 16: 3611-3616, 2018.

15. Livak KJ and Schmittgen TD: Analysis of relative gene expression data using real-time quantitative PCR and the 2(-Delta Delta C(T)) method. Methods 25: 402-408, 2001.

16. Cai H, Lin H, Cao W, Sun J, Huang Y and Fang Y: Downregulation of miR-519a predicts poor prognosis and contributes to tumor progression in gastric cancer. Oncol Res Treat 43: 19-26, 2020.

17. Chen S, Li F, Chai H, Tao X, Wang H and Ji A: miR-502 inhibits cell proliferation and tumor growth in hepatocellular carcinoma through suppressing phosphoinositide 3-kinase catalytic subunit gamma. Biochem Biophys Res Commun 464: 500-505, 2015.

18. Sun LL, Wang J, Zhao ZJ, Liu N, Wang AL, Ren HY, Yang F, Diao KX, Fu WN, Wan EH and Mi XY: Suppressive role of miR-502-5p in breast cancer via downregulation of TRAF2. Oncol Rep 31: 2085-2092, 2014.

19. Zhai H, Song B, Xu X, Zhu W and Ju J: Inhibition of autophagy and tumor growth in colon cancer by miR-502. Oncogene 32 : 1570-1579, 2013

20. Beishline K and Azizkhan-Clifford J: Sp1 and the 'hallmarks of cancer'. FEBS J 282: 224-258, 2015.

21. Chakraborty G, Rangaswami H, Jain S and Kundu GC: Hypoxia regulates cross-talk between Syk and Lck leading to breast cancer progression and angiogenesis. J Biol Chem 281: 11322-11331, 2006.

22. Zhang X, Jiang Y, Xie Y, Leng X, He M and Song F: Long noncoding RNA TRPM2-AS induced by SP1 inhibits cell apoptosis via MAPK and STAT3 in gastric cancer. J Gastroenterol Hepatol: May 18, 2020 (Epub ahead of print). 
23. Guan H, Cai J, Zhang N, Wu J, Yuan J, Li J and Li M: Sp1 is upregulated in human glioma, promotes MMP-2-mediated cell invasion and predicts poor clinical outcome. Int J Cancer 130: 593-601, 2012

24. Kou XX, Hao T, Meng Z, Zhou YH and Gan YH: Acetylated Spl inhibits PTEN expression through binding to PTEN core promoter and recruitment of HDAC1 and promotes cancer cell migration and invasion. Carcinogenesis 34: 58-67, 2013.

25. Lee HS, Park CK, Oh E, Erkin ÖC, Jung HS, Cho MH, Kwon MJ, Chae SW, Kim SH, Wang LH, et al: Low SP1 expression differentially affects intestinal-type compared with diffuse-type gastric adenocarcinoma. PLoS One 8: e55522, 2013.
26. Hsu TI, Wang MC, Chen SY, Yeh YM, Su WC, Chang WC and Hung JJ: Sp1 expression regulates lung tumor progression. Oncogene 31: 3973-3988, 2012.

This work is licensed under a Creative Commons Attribution-NonCommercial-NoDerivatives 4.0 International (CC BY-NC-ND 4.0) License. 